\title{
Evolution of supergaussian pulses in nonlinear Kerr media
}

\author{
Jerzy Jasiński, Łukasz Michalik \\ Faculty of Physics, Warsaw University of Technology, Koszykowa 75, 00-662 Warszawa
}

Received December 17, 2009; accepted December 30, 2009; published December 31, 2009

\begin{abstract}
The propagation of temporal pulses through nonlinear Kerr media with an initial supergaussian shape is described analytically and numerically. The analytical description is based on the canonical method. For a supergaussian profile as the trial function, the EulerLagrange equations are derived and solved. Accuracy of the canonical description and it's regime of applicability is discussed.
\end{abstract}

In Kerr media the envelope $U(z, t)$ of a pulse propagating along the $z$-direction satisfies the nonlinear Schrödinger equation (NSE) [1-3]:

$$
U_{z}+\frac{i k_{2} U_{t t}}{2}-i \varepsilon_{2}|U|^{2} U=0 .
$$

where $k_{2}<0$ is the group velocity dispersion coefficient, $\varepsilon_{2}>0$ is the nonlinear permittivity, and the indices $z$ and $t$ mean differentiation over those coordinates. Although the NSE has many exact solutions (e.g. solitons of many types), frequently it should be solved approximately or numerically to describe the evolution of a signal of a chosen shape, even in the case of a simple gaussian pulse.

One of most popular methods for obtaining approximate solutions of the NSE for a wide class of initial shapes is the variational (canonical) method [2-4]. In this paper this method is used to find an analytical description of the propagation of a supergaussian (SG) pulse:

$$
U(t, z)=b(z) \exp \left(-\frac{1}{2}\left|\frac{t}{w(z)}\right|^{2 m(z)}\right) .
$$

of any variable order $m(z)$ (for $m(z)=m_{0}=1$ SG pulse becomes gaussian). Although a pulse of shape (2) is a useful for approximating a single "bit" of information propagating in signals, it is rarely considered in the literature [2,5], especially in nonlinear cases. Reference [5] analyses SG pulses of variable order $m(z)$ and the effect of wave breaking occurring in highly nonlinear fibres. The method of moments was used in that work.

To describe the main features of SG pulse propagation Eq. (1) is solved numerically for an initial field (2). Such a numerical solution can be obtained by means of the FDBPM method [6]. In Fig.1 are shown two different solutions corresponding to the same pulse power, namely an initial gaussian pulse $m(0)=1$ and a supergaussian pulse of initial order $m(0)=2.5$. They produce two

${ }^{*}$ E-mail: jasinski@if.pw.edu.pl different scenarios of pulse propagation - infinite spreading in the left figure and oscillations in the right. These results depend only slightly on the initial pulse order - mainly they are determined by pulse's initial height and width.
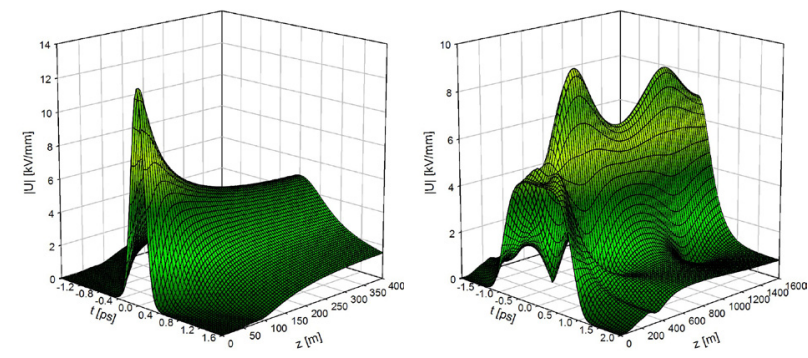

Fig. 1. SG pulse propagation in a nonlinear medium with $k_{2}=-1 \mathrm{ps}^{2} / \mathrm{km}$ and $\varepsilon_{2}=0.25 \mathrm{~nm} / \mathrm{kV}^{2}$. Left: initial height $b(0)=12.5 \mathrm{kV} / \mathrm{mm}$, initial width $w(0)=128 f s$, initial order $m(0)=1$, right: initial height $b(0)=5 \mathrm{kV} / \mathrm{mm}$, initial width $w(0)=800 \mathrm{fs}$, initial order 2.5. For both cases the initial phase profiles is $\varphi(0, t)=0$.

The important feature of propagation of both pulses considered in Fig. 1 is the change of their shape. It was found that after quite a short distance of propagation, the SG pulse, initially of order $m(0)=2.5$, evolves into a pulse better described by a lower order. Similar effects were observed for the gaussian pulse. In Fig. 2 we plot the pulse height, width and order as function of propagation distance $\mathrm{z}$ for an initial gaussian shape. The fitting was performed using the least squares method. The right figure shows an additional quantity - the standard deviation between the numerical solution and analytical approximation by a SG function of a variable order (pink curve) and the approximation by a gaussian function of constant order $m(z)=1$. This figure shows that the approximation based on a SG shape of variable order over the distance studied is 4 times better than the approximation using a gaussian function and the order itself decreases by more than a factor of two.

To describe the observed behaviour analytically let us write the Lagrange and Hamilton density corresponding to Eq. 1 [2-4]. Using an amplitude $r(z, t)$ and a phase $\varphi(z, t)$ instead of a complex amplitude $U(z, t)$ we have [7]: 


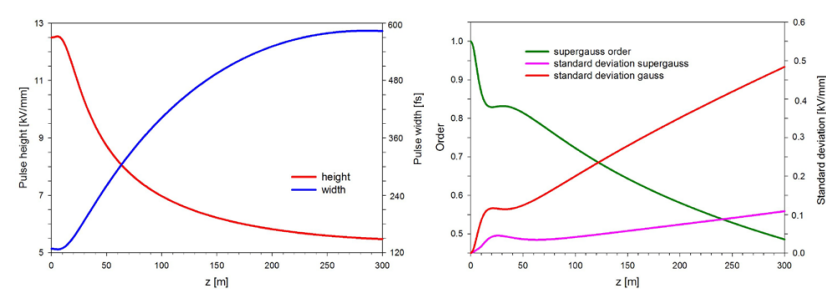

Fig. 2. Evolution of gaussian pulse parameters in anonlinear medium. Material parameters are the same as in Fig.1, pulse initial parameters as in the left part of Fig.1.

$$
\begin{gathered}
\mathcal{L}=r^{2} \varphi_{z}-\frac{k_{2}\left(r_{t}^{2}+r^{2} \varphi_{t}^{2}\right)}{2}-\frac{\varepsilon_{2} r^{4}}{2}, \\
\mathcal{H}=\frac{k_{2}\left(r_{t}^{2}+r^{2} \varphi_{t}^{2}\right)}{2}+\frac{\varepsilon_{2} r^{4}}{2} .
\end{gathered}
$$

A parabolic profile for the phase function $\varphi(z, t)$ with variable coefficient $\theta(z)$ describing its temporal curvature (chirp) is assumed. Moreover the real amplitude $r(z, t)$ is the product of a height function $b(z)$ and a shape function $g(t)$ (satisfying the condition $g(0)=1$ ). Assuming that $g$ depends on $z$ via the pulse width $w(z)$ and order $m(z)$ :

$$
\begin{gathered}
\varphi^{(s)}(z, t)=\beta(z)+t^{2} \theta(z), \\
r(z, t)=b(z) g\left((t / w(z))^{2 m(z)}\right) .
\end{gathered}
$$

Integrating the densities (3) over time from $-\infty$ to $+\infty$ the following lagrangian and hamiltonian were obtained:

$$
\begin{gathered}
\frac{L}{b^{2} w}=G_{1} \beta^{\prime}+G_{c} w^{2}\left(\theta^{\prime}-2 k_{2} \theta^{2}\right)-\frac{G_{k} k_{2}}{2 w^{2}}-\frac{\varepsilon_{2} G_{2} b^{2}}{2}, \\
\frac{H}{b^{2} w}=2 G_{c} k_{2} w^{2} \theta^{2}+\frac{G_{k} k_{2}}{2 w^{2}}+\frac{\varepsilon_{2} G_{2} b^{2}}{2},
\end{gathered}
$$

In the above expressions $G_{1}, G_{2}, G_{\mathrm{c}}$ and $G_{\mathrm{k}}$ are integrals of powers of the shape function and its derivatives:

$$
\begin{array}{cc}
G_{1}(x) & =\int_{-\infty}^{\infty} g\left(\tau^{2 x}\right)^{2} d \tau, \quad G_{2}(x)=\int_{-\infty}^{\infty} g\left(\tau^{2 x}\right)^{2} d \tau, \\
G_{c}(x) & =\int_{-\infty}^{\infty} \tau^{2} g\left(\tau^{2 x}\right)^{2} d \tau, \quad G_{c}(x)=x^{2} \int_{-\infty}^{\infty} \tau^{2(2 x-1)} g^{\prime}\left(\tau^{2 x}\right)^{2} d \tau .
\end{array}
$$

Notice, that the dependence of the lagrangian and hamiltonian on order $m$ is hidden in the coefficients (6) in which $x=m(z)$. For a SG shape $g(x)=\exp (-x)$ and all integrals (6) can be performed. The results of integration are expressed by the Euler gamma function $\Gamma(x)$ :

$$
\begin{array}{ll}
G_{1}(x)=2 \Gamma\left(1+\frac{1}{2 x}\right), & G_{2}(x)=2^{1-1 /(2 x)} \Gamma\left(1+\frac{1}{2 x}\right), \\
G_{c}(x)=\frac{2}{3} \Gamma\left(1+\frac{3}{2 x}\right), & G_{c}(x)=x \Gamma\left(1-\frac{1}{2 x}\right) .
\end{array}
$$

Any generalized coordinate $\xi$ satisfies the EulerLagrange equation $\partial L / \partial \xi-\partial / \partial z\left(\partial L / \partial \xi_{z}\right)=0$ [8]. For $\xi=\beta$ :

$$
G_{1} b^{2} w=P .
$$

Using the definition of $G_{1}, P$ is the power density:

$$
P=\int_{-\infty}^{\infty} r^{2}(z, t)=\operatorname{const}(z)
$$

Combining equations for $\xi=b$ and $\xi=w$ :

$$
\begin{gathered}
\beta^{\prime}=\frac{4 k_{2} G_{k}}{G_{1} w^{2}}+\frac{5 \varepsilon_{2} G_{2} b^{2}}{4 G_{1}}, \\
\theta^{\prime}-2 k_{2} \theta^{2}=-\frac{2 k_{2} G_{k}}{G_{c} w^{4}}-\frac{\varepsilon_{2} G_{2} b^{2}}{4 G_{c} w^{2}} .
\end{gathered}
$$

The remaining two Euler-Lagrange equations for $\xi=\theta$ and $\xi=m$ are more complicated. To simplify them, define the following reduced dimensionless width $J(z)$, and $N(z)$ and $D(z)$ as two combinations of the shape integrals (6):

$$
\begin{gathered}
J(z)=-\frac{\varepsilon_{2} P}{4 k_{2}} \sqrt{\frac{G_{c}(m(z))}{G_{1}(m(z))}} \cdot w(z), \\
N(z)=\frac{G_{k}(m(z)) G_{c}(m(z))}{G_{1}(m(z))^{2}}, \\
D(z)=\sqrt{\frac{G_{c}(m(z)) G_{2}(m(z))^{2}}{G_{1}(m(z))^{5}}} .
\end{gathered}
$$

Using the above definitions, the expressions are obtained:

$$
\begin{gathered}
\theta=-\frac{J^{\prime}}{2 k_{2} J}, \\
J=\frac{N^{\prime}}{D^{\prime}} .
\end{gathered}
$$

Since $N$ and $D$ defined in (11) depend on $z$ by means of $m$, the reduced width $J$ has the same property. Therefore by combining equations (8)-(12) it is possible to express four functions $\beta^{\prime}, \theta, w$ and $b$ in terms of the variable order $m(z)$. A fifth equation describes the evolution of the pulse order. It is a second order differential equation which is more complicated than the other equations. But it is possible to integrate it once to obtain the same expression as given by the law of conservation of canonical energy $H(z)=$ const (see [3] or [7]). The explicit form of equation resulting from this law of conservation is:

$$
\frac{8 k_{2}}{\varepsilon_{2}^{2} P}\left(\frac{d J}{d z}\right)^{2}+\frac{\varepsilon_{2}^{2} P^{3} N}{8 k_{2} J^{2}}-\frac{\varepsilon_{2}^{2} P^{3} D}{8 k_{2} J}=H_{0} .
$$

where $H_{0}$ is a value of the hamiltonian obtained from initial conditions. Interpreting the first term in the lefthand expression as a "kinetic energy", the sum of two remaining terms can be treated as potential $V(z)$ :

$$
V=-\frac{\varepsilon_{2}^{2} P^{3}}{8 k_{2}}\left(\frac{D}{J}-\frac{N}{J^{2}}\right) .
$$

Analogously to $N, D$ and $J$, the potential $V$ also depends on $z$ by means of a SG pulse of order $m$. Observe that a solution of Eq. (13) exists only for $H_{0} \leq V$. 
Since the potential $V$ is an explicit function of the pulse order, the solution of Eqn. (13) can be written in the form:

$$
z= \pm \sqrt{-\frac{8 k_{2}}{\varepsilon_{2}^{2} P}} \cdot \int \frac{J^{\prime}(m) d m}{\sqrt{V(m)-H_{0}}} .
$$

Having $m(z)$ allows the evolution of pulse width $w(z)$ and height $b(z)$ to be described by the following functions:

$$
\begin{gathered}
w(m)=-\frac{4 k_{2} J(m)}{\varepsilon_{2} P} \sqrt{\frac{G_{1}(m)}{G_{c}(m)}}, \\
b(m)=\sqrt{-\frac{\varepsilon_{2} P^{2}}{4 k_{2} J(m)} \sqrt{\frac{G_{c}(m)}{G_{1}(m)^{3}}}} .
\end{gathered}
$$

Unfortunately, the integration (15) required to obtain $m(z)$ cannot be performed analytically. Nevertheless, many features of the evolution can be surmised from an analysis of the potential and pulse width and height as function of the SG order $m$. In Fig. 3 we plot two of these functions:
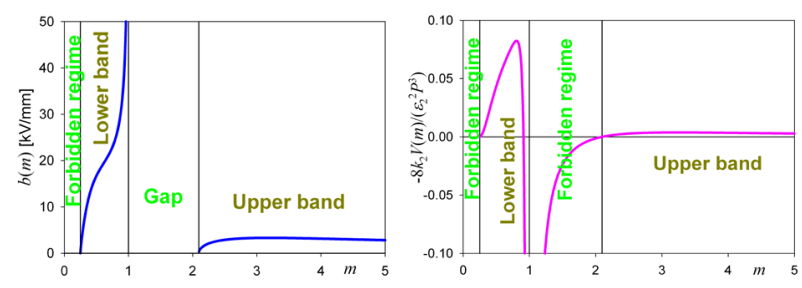

Fig. 3. Pulse height and potential vs. SG pulse order.

First note that for negative $\varepsilon_{2} / k_{2}$ the reduced width $J$ must be positive in order to obtain physical values of the height $b$. That is the reason why the function $b(m)$ is broken into two branches divided by the gap $1<m<$ 2.0963 where $J(m)<0$ (the forbidden regime for $\mathrm{m}<1 / 4$ appears because of the singularity in $\left.G_{\mathrm{c}}(1 / 4)\right)$. Note also that the heights of pulses in the lower branch are much smaller than those in the upper branch. Consequently, the lower band pulses have widths much larger than the upper band ones.

Since $H_{0}=$ const and $H_{0} \leq V$ during the evolution, the potential $V(m)$ changes between the initial value $V\left(m_{i}\right)$ and points at which $V(m)=H_{0}$ or the boundaries of the band. Therefore one should expect oscillations of all pulse parameters for positive $H_{0}$ and a monotonous change of them for negative $H_{0}$ (left and right profile illustrated in Fig. 1 correspond to negative and positive hamiltonians). Unfortunately, during propagation the deviation of the pulse from a SG shape increases (Fig. 2) and at large $z$ the description by the function (2) fails. Since deviations are much larger for the pulses of high initial order it is concluded that the method used can give a good description only for an initial order not greater than 1 and then only for a limited distance of propagation.

The left side of Fig. 4 shows the numerically computed SG order $m(z)$ given by the integral $(15)$ for $m(0)=0.98$.
The order decreases and values close to 0.25 are obtained at very large distances where the differences between the numerical and analytical profiles are quite significant. The right figure compares a few profiles of this pulse after propagation over the distance $z=300 \mathrm{~m}$ (for the assumed $k_{2}, \varepsilon_{2}$ and field initial values, this corresponds to about 11 nonlinear lengths). It appears that a SG function of variable order approximates the numerical profile much better than a function of constant order $m(z)=m(0)$. However its shape near the peak is visibly different. The fitting performed by the least squares method gives values $m(z), b(z)$ and $w(z)$ very close to values determined from (15) and (16), for example $m_{\text {fit }}=0.488$ and $m_{\text {Euler- }}$ Lagrange $=0.504$ (in Fig. 4 these two shapes are almost indistinguishable).
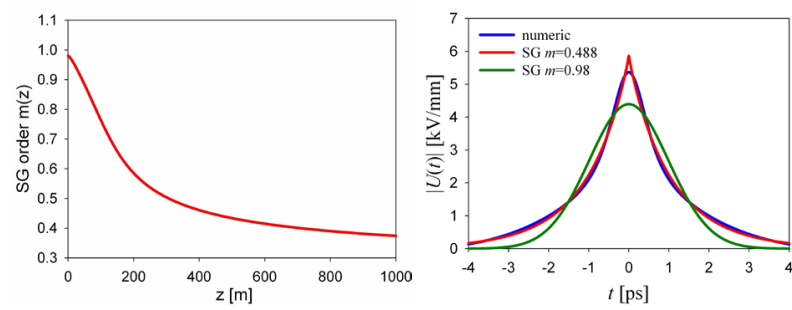

Fig. 4. Left: evolution of order described by an Euler-Lagrange equation. Right: comparison of numerical and analytic solutions.

To conclude, the canonical method applied to the propagation of SG pulses in Kerr nonlinear media gives a reasonably good analytical description when the order is less than 1 . The changes in the pulse shape observed during numerical investigations of propagation over distances up to 20 nonlinear lengths can be described approximately by changes in the SG pulse order. In fact in a Kerr medium, regardless of the initial shape, the pulse during propagation transforms into a soliton and for a SG pulse its intermediate states can be described approximately by changes of the SG order quite well. Such a similarity appears in the regime of pulse spreading. However, when a pulse oscillates the differences are much larger. For pulses with high initial order the method discussed for the description gives only qualitative results, because the shape deformation during propagation is much larger.

\section{References}

[1] G.P. Agrawal, Nonlinear Fiber Optics, (Academic Press, San Diego 2001)

[2] D. Anderson, Phys. Rev. A, 27, 3135, (1983)

[3] C. Sulem, P.L. Sulem, The Nonlinear Schrödinger Equation: SelfFocusing and Wave Collapse, (New York, Springer-Verlag, 1999)

[4] G.P. Agrawal, Lightwave technology:telecommunications systems, (Wiley Interscience, Hoboken, 2005)

[5] C.-J. Rosenberg, D. Anderson, M. Desaix, P. Johannisson, M. Lisak, Opt. Commun., 273, 272-277, (2007)

[6] J. Jasiński, Phot. Lett. Poland 1, 64, (2009)

[7] J. Jasiński, Phot. Lett. Poland 1, 139, (2009)

[8] H. Goldstein, C. Poole, J. Safko, Classical Mechanics, (Addison Wesley, San Francisco, 2000) 